\title{
Pseudohypopyon in a phakic patient following intravitreal injection of Triamcinolone Acetonide. Case report and review of Literature.
}

\author{
Dr.Pradeep AV ${ }^{1}$, Dr.Sonali Rao ${ }^{2}$, Dr.Sharan Shetty ${ }^{3}$, Dr.Talpallikar Neha ${ }^{4}$ \\ ${ }^{1}$ Assistant Professor,Department of Ophthalmology, Sri Dharmasthala Manjunatheshwara College of Medical \\ Sciences, India. \\ ${ }^{2,3,4}$ Post Graduate, Department of Ophthalmology, Sri Dharmasthala Manjunatheshwara College of Medical \\ Sciences, India.
}

\begin{abstract}
:
Purpose: Intravitreal Triamcinolone Acetonide (IVTA) is one of the treatment options for Macular Edema (ME). This can cause several complications like transient elevation of intraocular pressure, cataract formation and endopthalmitis. We describe an atypical complication of IVTA.

Case: A 60-year-old South Indian male presented with ME secondary to central retinal vein occlusion. We performed intravitreal injection of $4 \mathrm{mg}$ of Triamcinolone acetonide (TA). However, the drug dispersed behind equatorial part of posterior lens capsule and also into the anterior chamber.

Conclusion: Although IVTA is an easy and effective treatment for ME, TA may cause inadvertent complications when injected into the eye. Early recognition and appropriate management of such complications is essential.

Keywords: Intravitreal Injection, Pseudophyopyon, Triamcinolone Acetonide.
\end{abstract}

\section{Introduction}

Macular edema (ME) is caused by breakdown of the blood-retinal barrier which induces capillary leakage with accumulation of fluid in the intraretinal and subretinal spaces of the macula. Triamcinolone acetonide is a synthetic glucocorticoid used for its anti-inflammatory effect. It is crystalline in nature and disperses in the vitreous causing little or no reaction in the surrounding vitreous. IVTA is being used to treat a variety of ocular diseases such as cystoid macular edema after cataract surgery, macular edema due to inflammatory conditions, retinal vascular diseases like branch retinal vein occlusion (BRVO) and central retinal vein occlusion (CRVO)[1] and diabetic maculopathy[2] . Though IVTA is a simple and effective treatment, it sometimes causes complications. The common complications due to the drug itself are transient elevation of intraocular pressure and cataract[3]. Other complications may occur due to flawed intra vitreal injection techniques like retinal detachment, vitreous hemorrhage and endophthalmitis. Hence, to avoid such complications TA should be delivered to the main target, the posterior pole.

\section{Case Report}

A 60-year-old male presented to our clinic with decreased vision in his right eye from past six months. On examination his best-corrected visual acuity was $2 / 60$ in the right eye. Fundus examination revealed an old central retinal vein occlusion associated with ME. The patient was advised IVTA after explaining the risks and benefits of IVTA injection and an informed consent was taken. After following sterile aseptic precautions, $10 \%$ betadine was applied to the conjunctiva, lids, and lashes for ten minutes prior to the procedure. Preoperative anesthetic eye drops was instilled into the conjunctiva. A 30-gauge needle with $4 \mathrm{mg}(0.1 \mathrm{ml})$ of unfiltered TA was injected $3.5 \mathrm{~mm}$ posterior to the limbus into the mid-vitreous cavity under an operating microscope, and the tip of the needle was carefully positioned in the vitreous cavity during injection. Postoperatively, indirect ophthalmoscopy was used to examine the optic nerve for arterial pulsation, correct dispersion of the steroid and to rule out immediate complications, such as vitreous hemorrhage and retinal detachment. Intraocular pressure (IOP) measurement taken within 5 minutes of the procedure was recorded as $25 \mathrm{mmHg}$ with minimal pain. Topical antibiotic was prescribed postoperatively four times a day for three days as prophylaxis.

Patient was advised for follow-up next day, slit lamp examination revealed TA in the anterior chamber (Fig.1) and behind the posterior lens capsule (Fig.2). Patient's visual acuity decreased to $1 / 60$. Ten days after IVTA, TA persisted behind the lens and in the vitreous cavity. Although the amount of TA crystals behind the lens capsule decreased, the crystals persisted for more than 1 month. Only a small volume of TA was absorbed after 2 months, with final visual acuity of $3 / 60$ and IOP as $18 \mathrm{mmHg}$. Patient was suggested observation. We performed subsequent follow-up in the outpatient clinic with IOP measurement and deferred surgery. 


\section{Figures}

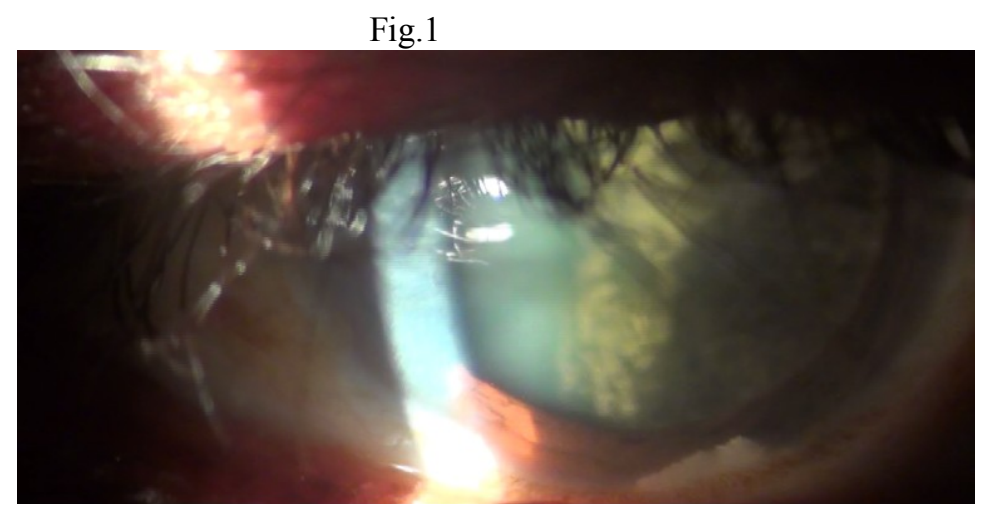

Right eye showing pseudohypopyon due to TA crystals in the anterior chamber.

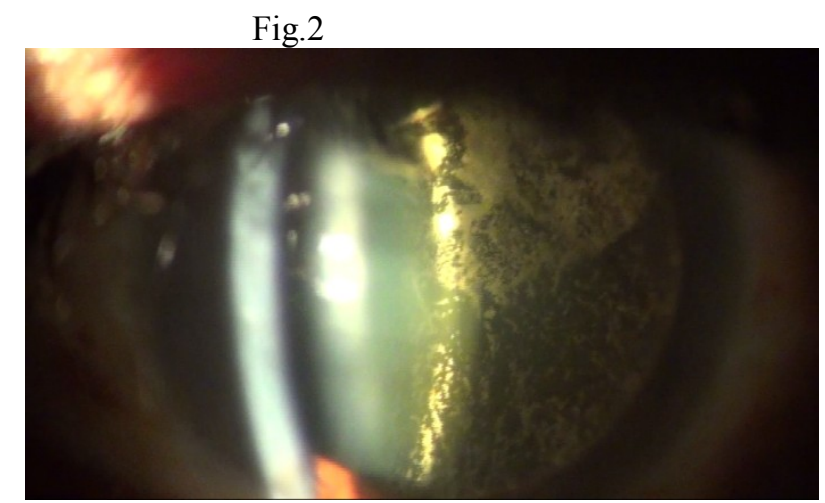

Right eye showing dispersed TA crystals on the posterior capsule.

\section{Conclusion}

Several reports have mentioned TA dispersion to the posterior lens capsule after injection. TA absorption is slow, resulting in prolonged loss of visual acuity due to disruption of the transparent vitreous [4,5]. Changes in the anterior vitreous, such as Berger's space, due to aging may cause such abnormal TA dispersion. Hosseini et al reported that TA localized in homogeneously in the vitreous area after injection in $53.6 \%$ of IVTA cases and that this TA persisted for up to 7 weeks after IVTA, indicating that several months are required for complete TA absorption[6] .In addition, subsequent fundus examinations were obscured, precluding detection of pathological events in the retina. However, in a few cases, 1 to 6 months were required for natural absorption, and surgical treatments were required in some cases[4,5].

The incidence of noninfectious endophthalmitis following IVTA injection ranges from $0.2 \%$ to $1.6 \%[7-10]$. Sutter et al[7] reported that 4 out of 600 patients $(0.6 \%)$ developed painless noninfectious endophthalmitis after IVTA injection. One of the four patients was treated with intravitreal antibiotic injections, but vitreous culture was negative. The other three were observed without progressive inflammation. Nelson et al[9] described noninfectious endophthalmitis after IVTA injection in 7 out of $440(1.6 \%)$ patients who were managed by observation without adverse outcomes. Jonas et al[10] reported that pseudohypopyon developed in 1 out of $454(0.2 \%)$ patients after IVTA injection $(25 \mathrm{mg})$. Anterior chamber paracentesis demonstrated TA crystals and no evidence of infectious endophthalmitis.

Though IVTA is an easy and effective treatment for ME, accurate injection of TA is sometimes difficult to achieve. Thus, it is important to deliver TA close to the main target that is posterior pole of the retina to prevent it from dispersing elsewhere. Injection under a surgical microscope is a much safer and effective approach.

Pseudohypopyon after IVTA injection may be managed initially with close observation[7-10]. In our case, clinical features of noninfectious endophthalmitis include absence of pain, eyelid edema and a normal fundus examination. Despite the probable noninfectious etiology, the clinical features of a true infectious endophthalmitis can be altered due the presence of steroids in the vitreous cavity. Therefore, these patients should be carefully followed to rule out progressive inflammation due to early endophthalmitis. 


\section{References}

[1] Williamson TH, O'Donnell A, Intravitreal triamcinolone acetonide for cystoid macular edema in non-ischemic central retinal vein occlusion, Am J Ophthalmol, 139, 2005, 860-6.

[2] Martidis A, Duker JS, Greenberg PB, Rogers AH, Puliafito CA, Intravitreal triamcinolone for refractory diabetic macular edema, Ophthalmology, 109, 2002, 920-7.

[3] J. B. Jonas, Intravitreal Triamcinolone Acetonide: A Change in a Paradigm, Ophthalmic Research, 38(4), 2006, $218-245$.

[4] A. Jain, M. R. Vishwanath and S. J. Charles, Triamcinolone Pseudo-Cataract. Annals of Ophthalmology, 38(1), 2006, 67-68.

[5] Y. B. Sakalar, K. Unlu, U. Keklikci, et al, Persistent Triamcinolone Acetonide particles on the posterior lens capsule after intravitreal injection, Annals of Ophthalmology, 40(3-4), 2008,190-192.

[6] H. Hosseini, H. R. Anvari-Ardakani, et al, Ultrasonographic pictures of Intravitreal Triamcinolone Acetonide, European Journal of Ophthalmology, 19(2), 2009, 263-267.

[7] Sutter FK, Gillies MC, Pseudo-endophthalmitis after intravitreal injection of triamcinolone, Br J Ophthalmol, 87, 2003, 972-974.

[8] Roth DB, Chieh J, Spirn MJ, et al, Non-infectious endophthalmitis associated with intravitreal triamcinolone injection, Arch Ophthalmol, 121, 2003, 1279-1282.

[9] Nelson ML, Tennant MT, Sivalingam A, et al, Infectious and presumed noninfectious endophthalmitis after intravitreal triamcinolone acetonide injection, Retina, 23, 2003, 686-691.

[10] Jonas JB, Kreissig I, Degenring RF, Endophthalmitis after intravitreal injection of triamcinolone acetonide, Arch Ophthalmol, 121, 2003, 1663-1664. 